\title{
Observation of surface morphology of plasma polymerized film by Atomic Force Microscope (AFM)
}

\author{
Daisuke Sato, Toshihiro Suwa, Masa-aki Kakimoto*, and Yoshio Imai \\ Department of Organic and Polymeric Materials, Tokyo Institute of Technology, \\ Meguro-ku, Tokyo 152, Japan
}

Keywords:plasma polymeriztion, D.C. glow discharge, AFM

\section{Introduction}

Thin films are readily obtained by plasma polymerization using various organic monomer gases. We have studied plasma polymerized films prepared under D.C. glow discharge. In this study, observation of surface of the plasma polymerized films prepared under several conditions by Atomic Force Microscope (AFM) is reported.

\section{Experimental}

Octamethylcyclotetrasiloxane (OMCTS) was supplied by Tokyo Kasei Co.. The plasma polymerized OMCTS films (PPOMCTS) was prepared by the plasma polymerization reactor utilized D.C. glow discharge as energy source supplied by Nippon Laser \& Electronics Lab. (Fig. 1). Mica was used as the substrate. Plasma polymerization was carried out as follows; First,

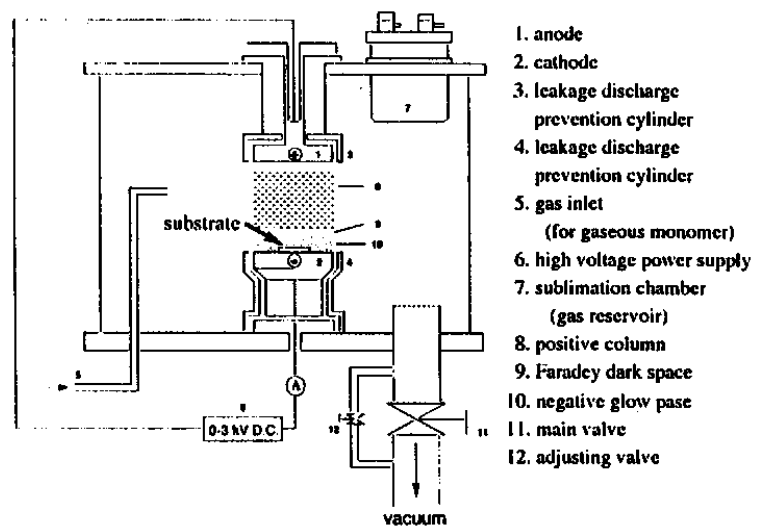

Fig.1 Plasma polymerization reactor utilized D.C. voltage the substrate was placed on the cathode and the reaction chamber was evacuated to below $10^{-2} \mathrm{~Pa}$. And then, OMCTS was introduced to the chamber via a sublimation chamber. A plasma polymerized film was formed on the substrate by application of the D.C.voltage. AFM was measured by using SPA 300 supplied by Seiko Instruments.

\section{Results and discussion}

We selected OMCTS as the monomer because of its adequate vapor pressure and high polymerization speed. Fig. 2 shows AFM images of PPOMCTS prepared under various gas pressures at the applied voltage of $0.8 \mathrm{kV}$. Minute grains are ovserved in every film.[1] When OMCTS gas pressure was increased, the diameter of the grains increased and their shapes became unclear. Fig. 3 shows film surface images in the case changing the applied voltage with keeping the gas pressure at $10 \mathrm{~Pa}$.

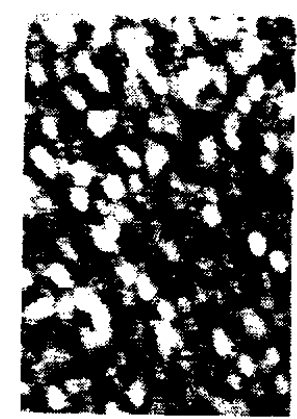

(a) $4 \mathrm{~Pa}$

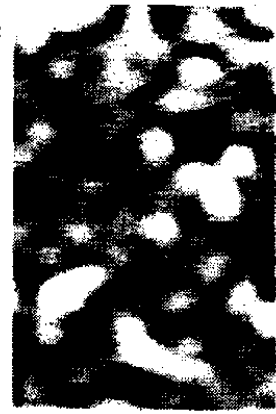

(b) $10 \mathrm{~Pa}$

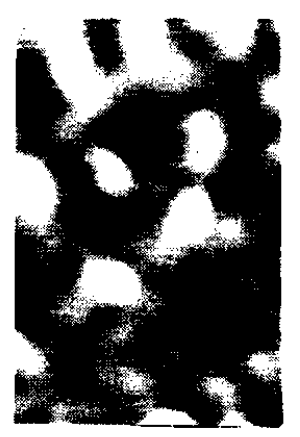

(c) $15 \mathrm{~Pa}$
Fig. 2 AFM images ofPPOMCTS at $0.8 \mathrm{kV}$ $100 \mathrm{~nm}$ 


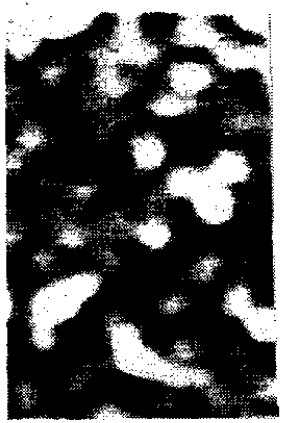

(a) $0.8 \mathrm{kV}$

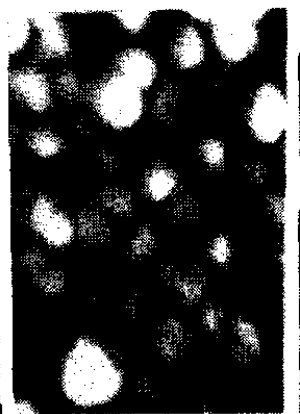

(b) $1.2 \mathrm{kV}$

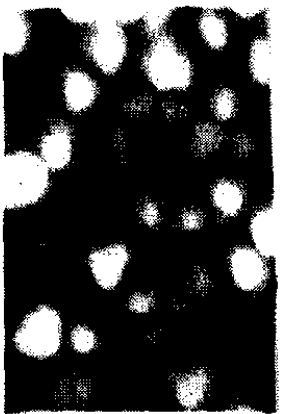

(c) $1.6 \mathrm{kV}$

Fig. 3 AFM images of PPOMCTS at 10Pa $\overleftrightarrow{100 \mathrm{~nm}}$

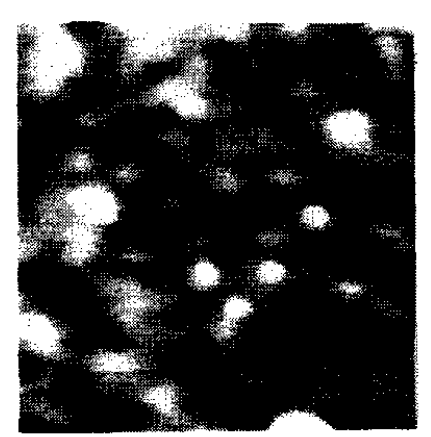

Fig. 4 AFM image of PPOMCTS at $0.8 \mathrm{kV}, 10 \mathrm{~Pa}$ prepared on the cathode $(500 \times 500 \mathrm{~mm})$

At the high voltage, the shapes of grains was clear. The surface observation of the samples prepared on the anode revealed the same grain structure as prepared on the cathode (Fig. 4). As the results of these observations, the following reaction scheme of PPOMCTS formation is proposed as shown in Fig. 5, in which two kinds of reaction processes occur simultaneously.[2, 3] (1) Agglomeration of activated monomer, then generation of three kinds of clusters which have positive charge, negative charge, and radical species, followed by deposition to the substrate. (2) Polymerization of the monomers on the substrate. As far as seeing the surface morphology of PPOMCTS, process (1) seems to be predominant compared with process (2) at lower gas pressure or the higher applied voltage, because the shape of grains became apparent under these conditions. Since these conditions made the energy given to every monomer larger, as the result, each monomer became more reactive to be easily agglomerated to the clusters, the surface structure formed like collection of clusters. On the contrary, when the

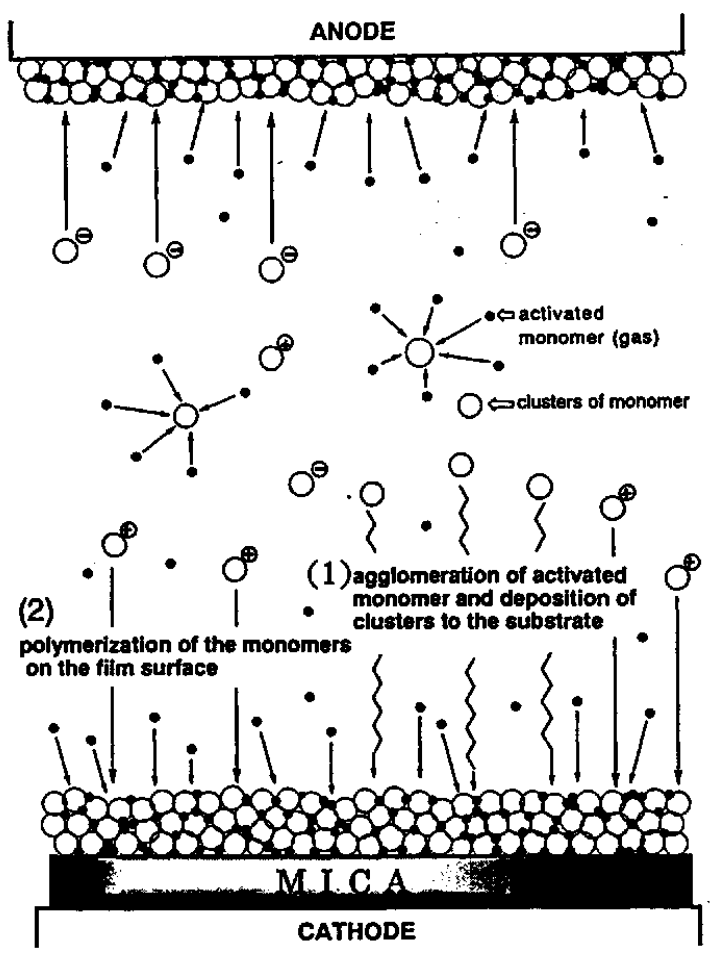

Fig. 5 The reaction scheme of PPOMCTS formation

gas pressure was increased, each monomer had less energy and the shape of grains was unclear because of process (2). In the case of the high applied voltage, ablation effect on the surface of the films was accelerated. Consequently only clusters remained at the surface of the film.

\section{Conclusion}

In growing process of plasma polymerized film, two processes occurs simultaneously; (1) Agglomeration of activated monomer, then generation of clusters followed by deposition to the substrate. (2) Polymerization of the monomers on the substrate. When the applied voltage is increased, generation of clusters and ablation effect were accelerated. Consequently surface structure of the film formed like collection of clusters.

\section{References}

1. M. Niinomi, H. Kobayashi, A. T. Bell, and M. Shen, J. Appl. Phys. , 44, 4317 (1973)

2. L. F. Thompson, K. G. Mayhan, J. Appl. Polym. Sci., 16, 2317 (1972)

3. H. Kobayashi, A. T. Bell, M. Shen, J. Appl. Polym. Sci., 17, 885 (1973) 